\title{
A Flexible Framework for Local Phase Coherence Computation
}

\author{
Rania Hassen, Zhou Wang, and Magdy Salama \\ Department of Electrical and Computer Engineering, University of Waterloo, \\ 200 University Avenue West, Waterloo, Ontario, Canada N2L 3G1 \\ raniahassen@ieee.org, zhouwang@ieee.org, M.Salama@ece.uwaterloo.ca
}

\begin{abstract}
Local phase coherence (LPC) is a recently discovered property that reveals the phase relationship in the vicinity of distinctive features between neighboring complex filter coefficients in the scale-space. It has demonstrated good potentials in a number of image processing and computer vision applications, including image registration, fusion and sharpness evaluation. Existing LPC computation method is restricted to be applied to three coefficients spread in three scales in dyadic scalespace. Here we propose a flexible framework that allows for LPC computation with arbitrary selections in the number of coefficients, scales, as well as the scale ratios between them. In particular, we formulate local phase prediction as an optimization problem, where the object function computes the closeness between true local phase and the predicted phase by LPC. The proposed method not only facilitates flexible and reliable computation of LPC, but also demonstrates strong robustness in the presence of noise. The groundwork laid here broadens the potentials of LPC in future applications.
\end{abstract}

Key words: local phase coherence, scale-space, complex wavelet coefficients, feature detection

\section{Introduction}

Phase information plays a crucial role in preserving important structural features in various types of signals, including 1D (e.g., speech), 2D (e.g., still images) and 3D (e.g., video or volume data) signals. For example, if the Fourier transform domain amplitude and phase spectra of two images are interchanged, the resulting hybrid image is recognized from which the phase spectrum is taken [1]. In understanding the structures of natural images, however, global Fourier phase may not be the best option, because natural images tend to be non-stationary, with different sizes and shapes of smooth or periodic regions, and distinctive features (such as edges and lines) between them. Furthermore, physiological studies suggest that many neurons located in the visual cortex are best models as filters localized in space, frequency and orientation. As a result, local phase is a more plausible quantity in cortical encoding and processing. In terms of image processing and understanding, it is also a better tool in describing the structures of natural images. 
In the pioneering work in studying the relation between congruence of local phases $[2,3]$, a local energy model was introduced which postulates that in a waveform which have unique perceptual significance as "lines" and "edges", the Fourier components come into phase with each other at these feature points. Based on this observation, it was suggested that the visual system could locate features of interest by searching for maxima of local energy points, and identify the feature type by evaluating the value of arrival or local phase at that point $[2$, $3]$. Almost all work thereafter concentrated on finding points of maximal phase congruency by looking for maxima in local energy. In [4], a direct measure of phase congruency was proposed, where a phase congruency measure is computed as a dimensionless quantity that is invariant to changes in image brightness or contrast and thus provides an absolute measure of the significance of feature points. Through the use of wavelets, an extension from 1D to 2D phase congruency calculation is also developed [4]. Local phase based method has also been employed in a number of computer vision and image processing problems, including estimation of image disparity [5] and motion [6][7], description of image texture [8], recognition of persons using iris patterns [9], and video quality assessment [10].

In [11], the local phase structures at distinctive features were examined in more depth. The local phase coherence (LPC) relationship was first discovered, which not only predicts the alignment of phases across scales at the location of features (as found in earlier work), but also describes the full structure of local phase pattern in scale-space in the vicinity of feature location. It was suggested in [11] that the LPC relation could lead to a new theory in the perception of blur, and may have deeper implications on how the visual system could "see beyond the Nyquist rate". Since the introduction of LPC, it has been found to be useful in a number of applications, including image registration [12], fusion [13] and sharpness evaluation [14]. One common limitation in all existing applications is that the LPC can only be computed with 3 coefficients spread in 3 scales in dyadic scale-space. This restricts its application, especially when one would like to have a closer examination of LPC relationship in the scale-space where smaller (and fractional) scale ratios are desired. The purpose of the current study is to develop a flexible methodology in computing LPC and thus extends its potentials in real applications.

\section{Local Phase Coherence and Computation}

\subsection{Local Phase Coherence}

The concept of LPC is built upon complex wavelet analysis tools that provide localized magnitude and phase information in multi-scales. Given a signal $f(x)$ localized near the position $x_{0}$, where $f(x)=f_{0}\left(x-x_{0}\right)$, a general complex wavelet transform may be written as:

$$
F(s, p)=\int_{-\infty}^{\infty} f(x) w_{s, p}^{*}(x) d x=\left[f(x) * \frac{1}{\sqrt{s}} g\left(\frac{x}{s}\right) e^{\jmath \omega_{c} x / s}\right]_{x=p},
$$




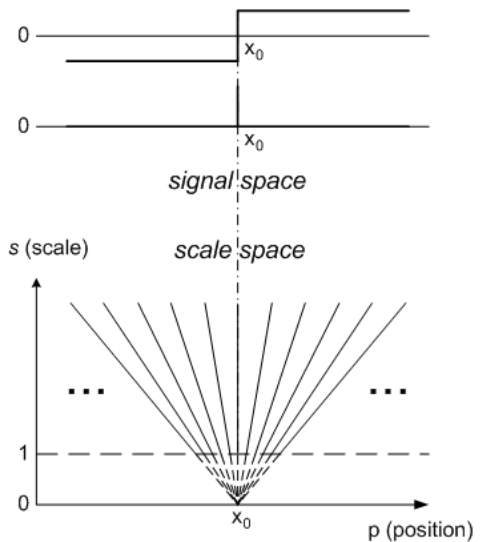

(a)

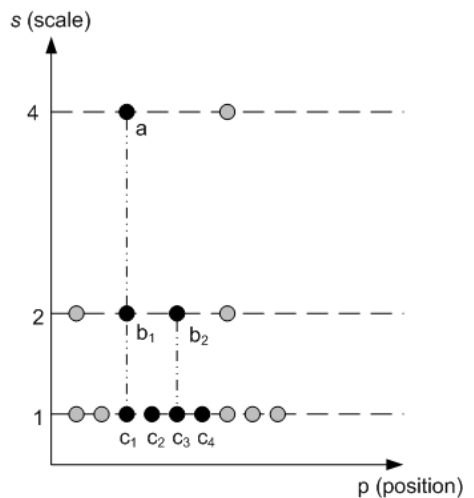

(b)

Fig. 1: (a) Local phase coherence structure near localized feature. (b) An example of $1 \mathrm{D}$ sampling grid in scale-space.

where $s \in R^{+}$is the scale factor, $p \in R$ is the translation factor, and the family of wavelets are derived from the mother wavelet $w(x)=g(x) e^{j \omega_{c} x}$ by

$$
w_{s, p}(x)=\frac{1}{\sqrt{s}} w\left(\frac{x-p}{s}\right)=\frac{1}{\sqrt{s}} g\left(\frac{x-p}{s}\right) e^{\jmath \omega_{c}(x-p) / s},
$$

where $\omega_{c}$ is the center frequency of the modulated band-pass filter, and $g(x)$ is a slowly varying and symmetric envelop function. Here the wavelet is considered general because we do not specify $g(x)$, which has many different options but the theory derived here applies to all.

Using the convolution theorem, and the shifting and scaling properties of the Fourier transform, we can derive:

$$
F(s, p)=\frac{1}{2 \pi \sqrt{s}} \int_{-\infty}^{\infty} F_{0}\left(\frac{\omega}{s}\right) G\left(\omega-\omega_{c}\right) e^{j \omega\left(p-x_{0}\right) / s} d \omega,
$$

where $F(\omega), F_{0}(\omega)$ and $G(\omega)$ are the Fourier transforms of $f(x), f_{0}(x)$ and $g(x)$, respectively. The phase of $F(s, p)$ depends on the nature of $F_{0}(\omega)$. If $F_{0}(\omega)$ is scale invariant, meaning that $F_{0}(\omega / s)=K(s) F_{0}(\omega)$, where $K(s)$ is a real function of only $s$, but independent of $\omega$, then it is not hard to find that:

$$
F(s, p)=\frac{K(s)}{\sqrt{s}} F\left(1, x_{0}+\frac{p-x_{0}}{s}\right) .
$$

Since both $K(s)$ and $s$ are real, we obtain the following phase relationship of $F(s, p)$ :

$$
\Phi(F(s, p))=\Phi\left(F\left(1, x_{0}+\frac{p-x_{0}}{s}\right)\right) .
$$


This result indicates that there is a strong phase coherence relationship across scale and space, where equal phase contours in the $(s, p)$ plane form straight lines that converge exactly at the location of the feature $x_{0}$, as illustrated in Fig. 1(a). These straight lines are defined by $x_{0}+\left(p-x_{0}\right) / s=C$, where $C$ is a constant. Note that this result is based on the assumption that $f_{0}$ is a scale invariant signal, which turns out to be true for distinctive features (such as an impulse or a step edge in a 1D signal, or an edge or line in a 2D image). Therefore, LPC measurement can be used to detect distinctive features in a signal.

\subsection{Computation of Local Phase Coherence}

If the LPC relationship is satisfied at a spatial location, then the phase of a wavelet coefficient may be predicted by the phases of its neighboring coefficients in the scale-space. Conversely, the prediction accuracy could be used as a measure of the strength of LPC. This approach was first employed in [11]. An example in shown in Fig. 1(b), where the finest scale coefficients $c_{i}$ for $i=1,2,3,4$ can be predicted from their coarser scale neighbors $a, b_{1}$ and $b_{2}$. For example,

$$
\hat{\Phi}\left(c_{1}\right)=-2 \Phi(a)+3 \Phi\left(b_{1}\right) .
$$

Although such prediction can lead to useful measures of the strength of LPC and has been successfully used in several applications [11-14], it is limited to grouping three coefficients at a time that are separated into three scales with fixed scale ratio of 2 between successive scales, as exemplified in Fig. 1(b). Here we propose a novel framework that allows for more flexibility in the computation of LPC. Let us consider a group of $N$ coefficients $a_{i}$ for $i=1, \ldots, N$, each of which is a sample of $F(s, p)$ at $\left(s_{i}, p_{i}\right)$, i.e., $a_{i}=F\left(s_{i}, p_{i}\right)$. If the LPC relationship is satisfied, then we should be able to best predict the phases of these coefficients, i.e., the error between the predicted and true phases should be minimized. The simplest form of an error function is the mean squared error

$$
E_{1}=\frac{1}{N} \sum_{i=1}^{N}\left(\Phi\left(a_{i}\right)-\hat{\Phi}\left(a_{i}\right)\right)^{2} .
$$

Note that for distinctive features such as a line or a step edge, the phase pattern in the scale-space can be approximated using a functional form. For example, in the case of a step edge $f_{0}(x)=K\left[u(x)-\frac{1}{2}\right]$, we have:

$$
\hat{\Phi}(F(s, p)) \approx \frac{w_{c}\left(p-x_{0}\right)}{s}-\frac{\pi}{2}+n_{1} \pi,
$$

where the constant term ( $-\frac{\pi}{2}$ here) depends on feature type, for another example, in the case $f_{0}(x)=K \delta(x)$, the constant is 0 . Assuming that a set of coefficients are aligned at the same position $p$ but across consecutive scales $s_{i}=1, r, r^{2}, \ldots . r^{N-1}$, where $r$ is the scale ratio between successive scales that may be any fractional number greater than 1 . Further, we simplify the phase 
prediction expression by denoting $Q_{p}=w_{c}\left(p-x_{0}\right)$. Then the problem of solving for best phase prediction is converted to

$$
Q_{p}^{(o p t)}=\arg \min _{Q_{p}} E_{1} .
$$

This can be solved by setting $\partial E_{1} / \partial Q_{p}=0$ and solve for $Q_{p}$, which leads to the following closed-form solution

$$
Q_{p}^{(o p t)}=\frac{\sum_{i=1}^{N} r^{(n-i)}\left(\Phi\left(a_{i}\right)+\frac{\pi}{2}\right)}{\sum_{i=1}^{N} r^{2(i-1)}} .
$$

In the case that the coefficients in scale-space are located at more than one position, say $M$, then we can solve for a series of $Q_{p}$ values $Q_{p_{1}}, Q_{p_{2}}, \ldots ., Q_{p_{M}}$ using similar approaches. After computing all $Q_{p}$ values, we will be able to calculate the predicted phases for all coefficients. We can then define an LPC measure as

$$
P C_{1}=\frac{\Re\left\{\prod_{i} a_{i} e^{-j \hat{\Phi}\left(a_{i}\right)}\right\}}{\prod_{i}\left|a_{i}\right|+C_{1}}=\frac{\Re\left\{\prod_{i}\left|a_{i}\right| e^{j\left[\Phi\left(a_{i}\right)-\hat{\Phi}\left(a_{i}\right)\right]}\right\}}{\prod_{i}\left|a_{i}\right|+C_{1}},
$$

where the numerator is the real part of the phase prediction error in the complex plane weighted by the coefficient magnitude, so that the coefficients with higher magnitudes are given more importance. The result is normalized by the magnitude of the coefficients. $C_{1}$ is a small positive constant in order to stabilize the measurement when the signal is close to flat, in which case the coefficients have near zero magnitudes. This measurement states that if the predicted phases are very close to the actual phases and the signal is significant (such that their magnitude is significantly larger than the constant $C_{1}$ ), then we achieve good LPC with a $P C_{1}$ value close to 1 . At the other extreme, if the predicted phases are perpendicular to the true phases, then the value of $P C_{1}$ will be close to 0 .

Although the above method and solution is elegant in the sense that it offers closed-form analytical solution, it does not give us satisfactory results in our experiments. This may be partially due to the $2 \pi$ wrap-around effect of angular variables (for which direct least square error function is deemed not appropriate). It may also be because the constant terms in the phase prediction form (e.g., Eq. (8)) varies for different types of features. For example, the $-\frac{\pi}{2}$ term in Eq. (8) would be $+\frac{\pi}{2}$ for a step edge $f_{0}(x)=K\left[\frac{1}{2}-u(x)\right]$ and 0 for an impulse $f_{0}(x)=K \delta(x)$.

To overcome the above problems, we define a new error energy function between the true and predicted phases as follows

$$
E_{2}=\left[1-\frac{1}{N} \sum_{i=1}^{N} \cos \left(4 \Phi\left(a_{i}\right)-4 \hat{\Phi}\left(a_{i}\right)\right)\right]^{2} .
$$

The trick here is to multiply the angles by a factor of 4 . This eliminates the ambiguities between the types of features because all the feature-dependent phase 
constants (such as the $-\frac{\pi}{2}$ term in Eq. (8)) are raised to a multiplier of $2 \pi$. In addition, the use of the cosine function in Eq. (12) avoids the $2 \pi$ wrap-around effect of angular variables. Notice that when the phase prediction is in effect, the difference $4 \Phi\left(a_{i}\right)-4 \hat{\Phi}\left(a_{i}\right)$ will be either close to 0 or a multiplier of $2 \pi$, and thus the cosine of it will be close to 1 . Consequently, the total error energy function $E_{2}$ will be close to 0 .

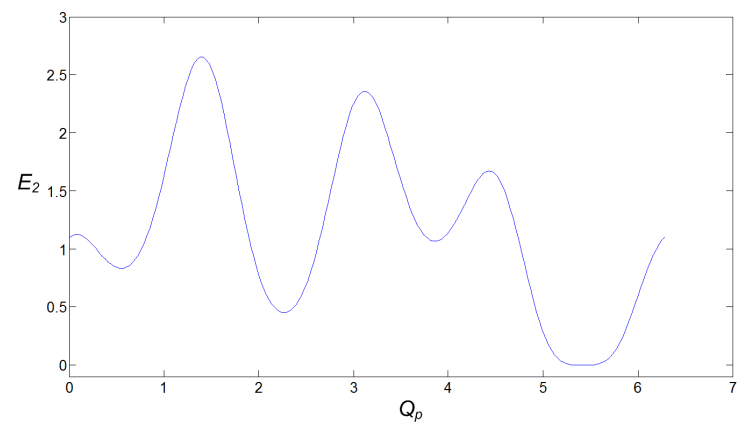

Fig. 2: An example of the search space of $E_{2}$ against $Q_{p}$.
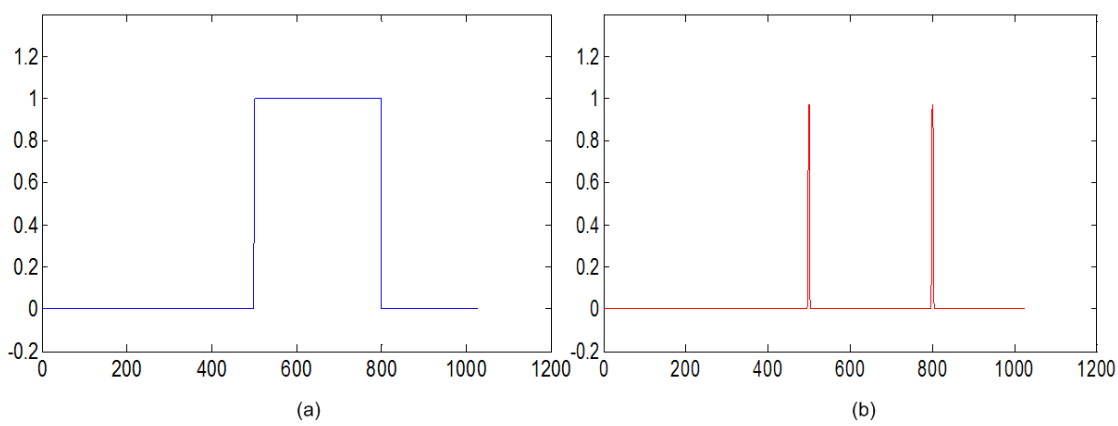

Fig. 3: (a) Original signal; (b) calculated LPC using Eq. (13)

Although the definition of $E_{2}$ has many good properties, it is a difficult function to optimize. For example, finding $Q_{p}$ using a closed-form solution like Eq. (10) is difficult. Indeed, $E_{2}$ could be a fairly complicated function. An example is shown in Fig. 2, where the function of $E_{2}$ with respect to $Q_{p}$ is smooth but has many local minima. In our implementation, we use an iterative numerical method to minimize the function, where the full search range is divided into 8 equally spaced segments, each associated with a different initial point at the 
center of the segment as the initial guess in the iteration. This results in multiple local minima, and then the global minimum is obtained by picking the lowest local minima. Finally, the LPC is computed by

$$
P C_{2}=\frac{\Re\left\{\prod_{i}\left(a_{i}\right)^{4} e^{-j 4 \hat{\Phi}\left(a_{i}\right)}\right\}}{\prod_{i}\left|\left(a_{j}\right)^{4}\right|+C_{2}}=\frac{\Re\left\{\prod_{i}\left|\left(a_{i}\right)\right|^{4} e^{j 4\left(\Phi\left(a_{i}\right)-\hat{\Phi}\left(a_{i}\right)\right)}\right\}}{\prod_{i}\left|\left(a_{j}\right)\right|^{4}+C_{2}} .
$$

Similar to Eq. (11), $C_{2}$ is a positive stabilizing constant, and this is an energy weighted phase consistency measure, where the maximal value is achieved if all phase predictions are perfect. Fig. 3(a) shows a simulated signal with ideal step edges, and Fig. 3(b) gives the LPC computation result using Eq. (13). It can be seen high $\mathrm{PC}_{2}$ values are achieved (high peaks) at the step edges.

\section{Simulations}

In this section we will present several experiments meant to gauge the performance and robustness of the proposed technique for LPC computation. Although the experiments were carried out in 1D (which helps us better visualize the performance of the algorithm), similar techniques can also be applied to $2 \mathrm{D}$ or higher dimensional signals.

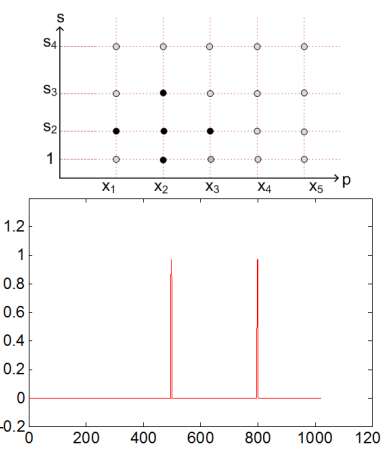

(a)
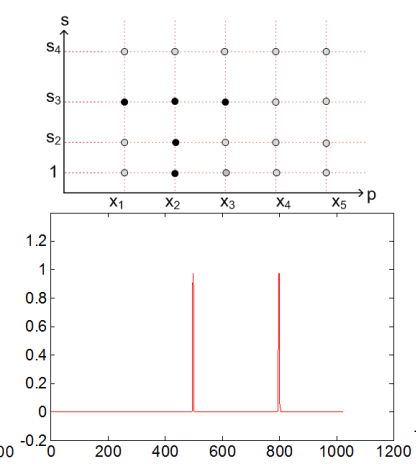

(b)

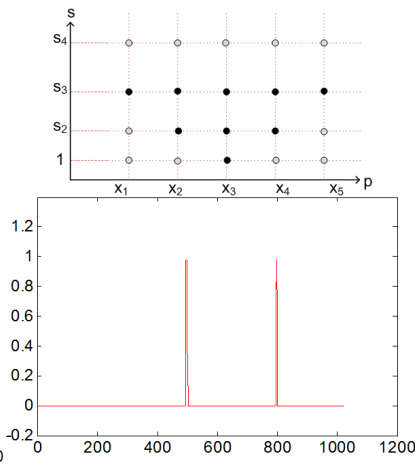

(c)

Fig. 4: LPC computation by grouping local coefficients in three different ways.

The first experiment aims to demonstrate the flexibility of our framework in picking arbitrary group of neighboring coefficients in LPC computation. The upper figures in Fig. 4 show three different selections of complex wavelet coefficients in the scale-space, where the coefficients spread in three scales and up to five spatial locations. The scale ratios between successive scales are fixed at 2 . The lower figures show the LPC measure $P C_{2}$ computed as a function of space 
for the signal in Fig. 3(a). Despite the quite different coefficient grouping, it can be observed that the resulting phase coherence functions are approximately the same. This result suggests that in practice, LPC can be computed in the complex wavelet transform domain with any coefficient setup, and may also be useful in the applications where only partial information of the local phase measurement is available.

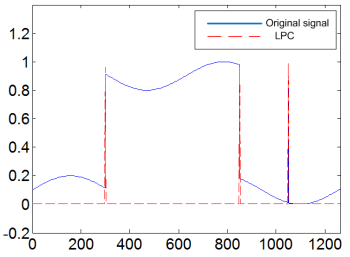

(a) $r=1.223$

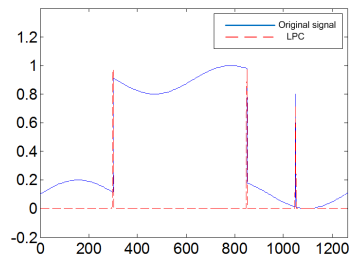

(b) $r=1.414$

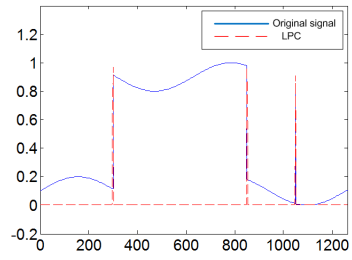

(c) $r=1,1.4,1.2$

Fig. 5: LPC computation for a signal by using fractional scale ratios between coefficients. (a) Fixed scale ratio of $r=1.223$ between 3 consecutive scales; (b) Fixed scale ratio of $r=1.414$ between 3 consecutive scales; (c) Varying scale ratio $r=1.4$ between the first 2 scales and $r=1.2$ between the second and third scales.

The second experiment demonstrates the flexibility in picking scale ratios between successive scales. Most existing wavelet transforms were designed in dyadic scale-space, i. e., the scale ratio between successive scales is fixed at 2 . From the derivations in the last section, this should not be a necessary condition in the computation of LPC. The scale ratio can be any other fractional number greater than 1. Even further, the scale ratio does not have to be the same between Scales 1 to 2 and Scales 2 to 3 . Figure 5 shows the resulted phase coherence using different setup of scale ratios. In the first two example, the scale ratios are fixed across three scales but are fractional numbers of $r=1.223$ and $r=1.414$, respectively. In the third example, the scale ratio is varying between the first two scales $r=1.4$ and the last two scales $r=1.2$. In all three cases, the resulting $P C_{2}$ functions are almost the same when applied to the same signal. This is a useful feature in practical applications because real world signals often contain mixtures of many distinctive features, and thus local measurement up to coarse scales often suffers from interference from nearby features. If the scale ratios can be fractional (preferably less than 2), then we will be able to carry out closer scale-space analysis of local features and avoid interference from nearby features.

The last experiment is concerned about the impact of noise on our LPC computation. Figure 6 shows the $P C_{2}$ function computed for a signal contaminated with additive white Gaussian noise at three noise levels. It can be seen that the LPC computation successfully detects the distinctive features (edges 


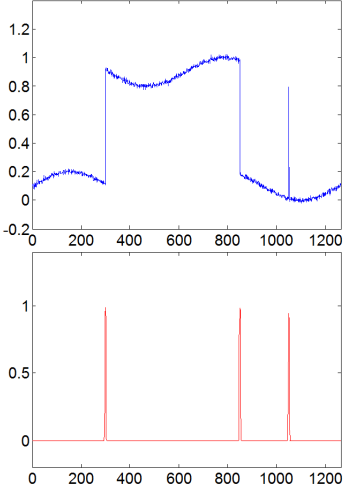

(a) $\sigma_{n}=0.01$

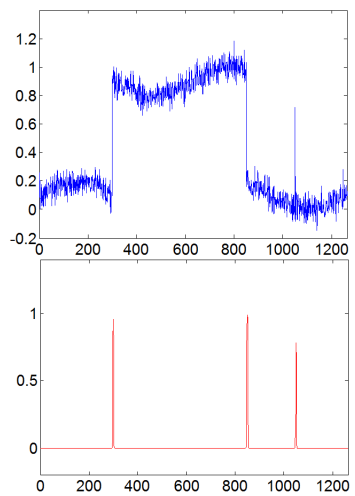

(b) $\sigma_{n}=0.05$

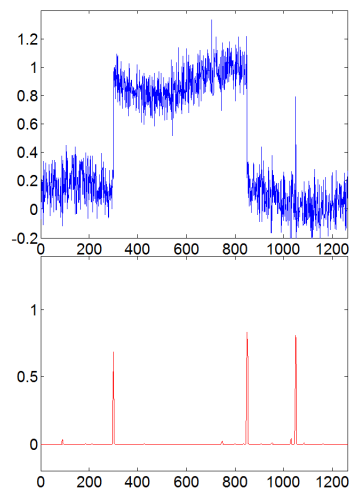

(c) $\sigma_{n}=0.1$

Fig. 6: LPC computation in the presence of additive white Gaussian noise, with noise standard deviation equaling (a) $\sigma_{n}=0.01$, (b) $\sigma_{n}=0.5$, and (c) $\sigma_{n}=0.1$.

and impulse) in all three cases, showing its strong robustness to noise (though the heights of LPC values may be moderately affected by heavy noise). This is another useful feature in practical applications, where many other techniques (e.g., derivative or gradient based edge detectors) are often sensitive to noise contaminations.

\section{Conclusion}

The purpose of this work is to extend the theory and methodology of local phase coherence, so that it can be converted to more practical techniques that can be applied to various signal processing applications for the analysis of signals and the detection of features. The major contribution of the current work as opposed to existing LPC computation is to formulate the problem using an optimization framework. Several technical issues have been studied in order to overcome a series of problems encountered in formulating the optimization problem and in finding the optimal solutions. The resulting LPC computation exhibits significantly broadened flexibilities such that it can be computed with arbitrary grouping of neighboring complex wavelet coefficients spread at any fractional scale ratios between successive scales. It also demonstrates strong robustness to noise. These flexibilities make our approach desirable in many potential applications, especially in the cases when multiple features exist and are close to each other, when only partial information of local phases is available, and/or when significant noise exists in the signal. Our future work is to apply the methodology developed in this work to practical signal and image applications, such as those in [12-14], so as to better exploit the advantages of LPC. 


\section{Acknowledgment}

This research was supported in part by Natural Sciences and Engineering Research Council of Canada in the forms of Discovery, Strategic and CRD Grants, and by an Ontario Early Researcher Award, which are gratefully acknowledged.

\section{References}

1. Oppenheim, A.V. and Lim, J.S: The importance of phase in signals. Proceedings of the IEEE, vol. 69, no. 5, pp. 529-541, (1981).

2. Morrone, M.C. and Burr, D.C.: Feature detection in human vision: a phasedependent energy model. Proceedings of the Royal Society of London, Series B, vol. 235 , no. 128 , pp. 221-245, (1988).

3. Morrone, M.C. and Owens, R.A.: Feature detection from local energy. Pattern Recognition Letters, vol.6, Issue 5, pp. 303-313, (1987).

4. Kovesi, P.: Image features from phase congruency. Journal of Computer Vision Research, vol.1, no. 3, pp. 1-26, (1999).

5. Fleet, D. J. : Phase-based disparity measurement. CVGIP: Image Understanding, vol. 53, no. 2, pp. 198-210, (1991).

6. Fleet, D. J. and Jepson, A. D.: Computation of component image velocity from local phase information. International Journal of Computer Vision, vol. 5, no. 1, pp. 77-104, (1990).

7. Wang Z. and Li Q. : Statistics of natural image sequences: temporal motion smoothness by local phase correlations. Human Vision and Electronic Imaging XIV, Proc. SPIE, vol. 7240, Jan. 19-22, (2009).

8. Portilla J. and Simoncelli, E. P. : A Parametric Texture Model based on Joint Statistics of Complex Wavelet Coefficients. International Journal of Computer Vision, vol. 40, pp. 49-71, (2000).

9. Daugman, J. : Statistical richness of visual phase information: update on recognizing persons by iris patterns. International Journal of Computer Vision, vol.45, no. 1, pp. 25-38, (2001).

10. Zeng K. and Wang Z. : Quality-aware video based on robust embedding of intraand inter-frame reduced-reference features. IEEE International Conference on Image Processing, Hong Kong, China, Sept. 26-29, (2010).

11. Wang, Z., Simoncelli, E.P.: Local phase coherence and the perception of blur. In Adv. Neural Information Processing Systems NIPS03, MIT Press, pp. 786-792, (2004).

12. Hassen R., Wang Z. and Salama M. : Multi-sensor image registration based-on local phase coherence. IEEE International Conference on Image Processing, Cairo, Egypt, Nov. 7-11, (2009).

13. Hassen R., Wang Z. and Salama M. : Multifocus image fusion using local phase coherence measurement. International Conference on Image Analysis and Recognition, Halifax, Canada, July 6-8, (2009).

14. Hassen R., Wang Z. and Salama M. : No-reference image sharpness assessment based on local phase coherence measurement. IEEE International Conference on Acoustics, Speech, and Signal Processing, Dallas, TX, Mar. 14-19, (2010). 